\title{
The Arabidopsis homolog of human G3BP1 is a key regulator of stomatal and apoplastic immunity
}

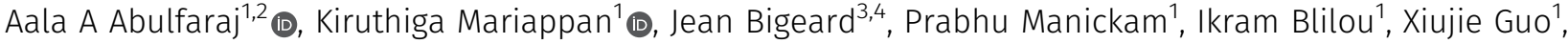 \\ Salim Al-Babili ${ }^{1}$ (D), Delphine Pflieger ${ }^{5,6}$ (D), Heribert Hirt ${ }^{1,3,4}$ (D), Naganand Rayapuram ${ }^{1}$ (1)
}

\begin{abstract}
Mammalian Ras-GTPase-activating protein SH3-domain-binding proteins (G3BPs) are a highly conserved family of RNA-binding proteins that link kinase receptor-mediated signaling to RNA metabolism. Mammalian G3BP1 is a multifunctional protein that functions in viral immunity. Here, we show that the Arabidopsis thaliana homolog of human G3BP1 negatively regulates plant immunity. Arabidopsis g3bp1 mutants showed enhanced resistance to the virulent bacterial pathogen Pseudomonas syringae pv. tomato. Pathogen resistance was mediated in Atg3bp1 mutants by altered stomatal and apoplastic immunity. Atg3bp1 mutants restricted pathogen entry into stomates showing insensitivity to bacterial coronatine-mediated stomatal reopening. AtG3BP1 was identified as a negative regulator of defense responses, which correlated with moderate up-regulation of salicylic acid biosynthesis and signaling without growth penalty.
\end{abstract}

DOI 10.26508/Isa.201800046 | Received 6 March 2018 | Revised 21 May 2018 | Accepted 22 May 2018 | Published online 31 May 2018

\section{Introduction}

RNA-binding proteins (RBPs) play a critical role in the regulation of gene expression, a feature that relies on their conformational plasticity and their capacity to interact with distinct targets (Jonas \& Izaurralde, 2013; Castello et al, 2016). In mammals, Ras-GTPaseactivating protein SH3-domain-binding protein 1 (G3BP1) belongs to a family of RBPs (Tourriere et al, 2001) that is located in stress granules (SGs) and contributes to their assembly (Tourriere et al, 2003). SGs are cytoplasmic aggregates containing stalled preinitiation complexes, which are thought to serve as sites of mRNA storage during the cell stress response (Anderson \& Kedersha, 2008). Cells lacking G3BP1 are compromised to form $\mathrm{SGs}$ in response to eukaryotic initiation factor $2 \alpha$ phosphorylation or eIF4A inhibition but are SG competent upon heat or osmotic stress. G3BP1 interacts with 40 S ribosomal subunits through the RGG motif, which is also required for G3BP-mediated SG formation
(Kedersha et al, 2016). G3BP1 is also involved in virus multiplication and modulates initiation of translation (Katsafanas \& Moss, 2007; White et al, 2007; Panas et al, 2012). G3BP1 is a multifunctional protein that is highly conserved in all eukaryotes, but so far, only one protein AtG3BP-like protein (At5g43960) was identified as an Arabidopsis thaliana G3BP-like protein that localizes to plant SGs and plays a role in the Arabidopsis virus resistance (Krapp et al, 2017). Here, we identify the Arabidopsis G3BP1 homolog that belongs to a family of eight proteins and functions in plant immunity.

As in mammals, plants protect themselves against a wide variety of invading pathogens with different resistance strategies. This is accomplished by a sophisticated multilayered plant innate immune system (Dangl \& Jones, 2001). Plants possess integrated immune signaling networks to facilitate rapid defense responses upon pathogen recognition, which in turn, restrict the further growth and spread of pathogens. In Arabidopsis, pathogen attack or treatment with a microbe-associated molecular pattern (MAMP), such as flg22 (the conserved 22-amino acid peptide derived from Pseudomonas aeruginosa flagellin), leads to the recognition of the MAMP by pattern recognition receptors that activate MAMP-triggered immunity (MTI). Receptor-mediated recognition of MAMPs leads to the induction of a battery of defense responses that include production of reactive oxygen species (ROS), an increase in intracellular calcium concentration ( $\mathrm{Yu}$ et al, 2017), and activation of several Arabidopsis mitogen-activated protein kinases (MAPK), including MPK3, MPK4, MPK6, and MPK11 (Zipfel et al, 2006; Ranf et al, 2011; Frei dit Frey et al, 2014). These early events after MAMP perception in turn lead to intermediate defense responses mediated by transcriptional reprogramming of defense-related genes (Zipfel et al, 2004); production of antimicrobial compounds, including phytoalexins such as camalexin; callose deposition; biosynthesis of stressrelated hormones such as salicylic acid (SA), jasmonic acid, and ethylene (Yu et al, 2017); and production of pathogen-related proteins such as PR1 (van Loon et al, 2006).

Recognition of MAMPs by pattern recognition receptors also triggers stomatal closure (Melotto et al, 2006; singh \& Zimmerli,

${ }^{1}$ Desert Agriculture Initiative, King Abdullah University of Science and Technology, Thuwal, Saudi Arabia ${ }^{2}$ Department of Biology, Science and Arts College, Rabigh Campus, King Abdulaziz University, Jeddah, Saudi Arabia ${ }^{3}$ Institute of Plant Sciences Paris-Saclay, CNRS, INRA, Université Paris-Sud, Université Evry, Université Paris-Saclay, Orsay, France ${ }^{4}$ Institute of Plant Sciences Paris-Saclay, Paris Diderot, Sorbonne Paris-Cité, Orsay, France ${ }^{5}$ Université Grenoble Alpes, CEA, Inserm, BIG-BGE, Grenoble, France

${ }^{6}$ CNRS, BIG-BGE FR3425, Grenoble, France

Correspondence: heribert.hirt@kaust.edu.sa 
2013). Stomata are natural openings in the plant that are used by microbial pathogens for entry into the plant. However, these organs are essential for plant survival as they allow gaseous exchange and transpiration. Stomata are critical during both biotic and abiotic stress responses (Acharya \& Assmann, 2009). The plant innate immune system limits infection by preventing the invasion of the apoplastic space and by compromising the proliferation of the pathogen postinvasion. Pseudomonas syringae secretes the phytotoxin coronatine (COR) that interferes with plant stomatal immunity (Melotto et al, 2006). In addition to disabling stomatal immunity, COR also inhibits apoplastic immunity through suppression of SA-mediated defenses (Zheng et al, 2012; Geng et al, 2014). SA is one of the key regulators of stomatal opening and closing. SA is a defense signal molecule against biotrophic and hemibiotrophic pathogens and regulates plant immune responses against several pathogens (Love et al, 2007; Chen et al, 2009; Wang et al, 2013; Ding et al, 2014). MAMPs also have been shown to induce SA accumulation (Mishina \& Zeier, 2007; Tsuda et al, 2008). SA synthesis and signaling pathways are necessary for bacterial and MAMP-induced stomatal closure in Arabidopsis (Melotto et al, 2006; Zeng et al, 2010). SA enhances defense signaling that leads to transcriptional reprogramming (Moore et al, 2011) and ROS accumulation (Sato et al, 2010; Xu et al, 2014). Furthermore, Arabidopsis mutants such as acd (accelerated cell death) and ssi (suppressor of salicylate insensitivity of npr1-5), which express high levels of SA, are shown to be more resistant to pathogen infection and constitutively express defense genes including $P R$ genes. In contrast, mutants with impaired SA biosynthesis such as sid2 (salicylic acid inductiondeficient-2) and eds5 (enhanced disease susceptibility-5) enhance disease susceptibility to various pathogens (Ding et al, 2014). Mutations in SA accumulation or signaling genes and Arabidopsis transgenic plants with the bacterial gene nah $G$ that encodes an SA hydrolase were compromised in defense against $P$. syringae pv. tabaci, Phytophthora parasitica, or Cercospora nicotianae (Delaney et al, 1994).

In this study, we characterize G3BP1 that belongs to a family of eight proteins in Arabidopsis. By studying loss-of-function mutants, we show that G3BP1 plants are resistant to Pst infection, have higher levels of $\mathrm{SA}$, and exhibit closed stomata and constitutive defense gene expression. In addition, overexpressor lines exhibited stomatal opening as in the WT but were more susceptible to $P$. syringae infection. Our results establish that AtG3BP1 negatively regulates plant stomatal and apoplastic immunity via SA-mediated defense.

\section{Results}

\section{AtG3BP1 is a homolog of the human HsG3BP1}

Human G3BP1 protein contains two well-characterized domains, namely the nuclear transport factor 2 domain and an RNA recognition motif. We searched the TAIR10 database for all proteins with these two conserved domains. The Arabidopsis genome codes for eight proteins which satisfy these criteria. The eight Arabidopsis G3BPs are named AtG3BP1 to AtG3BP8 as indicated in Fig S1A. The independent identification of different members of the AtG3BP family by several groups and the use of different naming systems have presented conflicts in the available literature, and a unified classification of AtG3BPs is desirable for further studies. A multiple alignment and phylogenetic tree for the relationship of G3BPs in Arabidopsis and human was generated using Molecular Evolutionary Genetics Analysis Version 7 (MEGA7) based on the amino acid sequences (Fig 1A) (Kumar et al, 2016). The phylogenetic tree was reconstructed using the neighbor-joining method (Saitou \& Nei, 1987). Spliced isoforms from a single gene were eliminated in the phylogenetic tree for simplicity. AtG3BP1 is a homolog to human HsG3BP1 with an amino acid identity of 32\% (Fig S1B).

\section{Atg3bp1 mutant lines show a normal developmental phenotype}

Two T-DNA insertion lines in the gene AT5G48650 were obtained from the National Arabidopsis Stock Center (NASC), Atg3bp1-1 (SAIL_1153_H01) and Atg3bp1-2 (SALK_027468). Using allele-specific primers, homozygous Atg3bp1-1 and Atg3bp1-2 mutants were identified and the site of insertion was determined by sequencing. In Atg3bp1-1, the T-DNA was inserted in the $5^{\text {th }}$ exon $844 \mathrm{bp}$ downstream of the ATG start site. In Atg3bp1-2, the T-DNA was inserted in the $9^{\text {th }}$ exon 1,280 bp downstream of the ATG start site (Fig 1C). Determination of the relative transcript levels by qPCR indicated that both Atg3bp1-1 and Atg3bp1-2 were loss-of-function mutants (Fig 1D). For overexpression studies, a cauliflower mosaic virus 355 promoter-AtG3BP1-GFP construct was introduced into Col-0 WT background to give homozygous overexpression lines OE2 and OE3. OE2 and OE3 showed higher expression levels of AtG3BP1 transcripts compared with WT controls (Fig 1E). Neither the loss-of-function mutants nor the overexpression lines showed visible growth or developmental phenotypes (Fig 1F).

\section{AtG3BP1 is expressed and localized to different cellular compartments}

To study the in planta expression of AtG3BP1, stably transformed promoter-GUS ( $\beta$-glucuronidase) reporter lines were produced. GUS analysis was performed on seedlings of 8-14 $d$ and staining was carried out from 1 to $12 \mathrm{~h}$. AtG3BP1 was expressed in all tissues under these conditions (Fig 2A).

We then assessed the subcellular localization using several in silico prediction tools, where AtG3BP1 was predicted to potentially sort to the chloroplast and nucleus (Fig S2). To validate these predictions, we expressed an AtG3BP1-GFP fusion protein under the cauliflower mosaic virus $35 \mathrm{~S}$ promoter in Arabidopsis stable lines (OE2) (Fig 2B). Confocal images indicated that AtG3BP1 is localized to various compartments within the cytoplasm (Fig 2C). We see expression of the recombinant protein in the cytoplasm of stomata in the hypocotyl region and true leaves (Fig 2C, ii-iv).

We also examined the subcellular localization of GFP-tagged AtG3BP1 transiently expressed in Nicotiana benthamiana leaf epidermal cells. Coinfiltration of vectors expressing AtG3BP1-GFP or Serrate-CFP, which served as internal reference marker for the nuclear compartment, showed that AtG3BP1-GFP was localized to the cytoplasm (Fig 2D).

\section{Loss of AtG3BP1 alters classical MTI responses}

To evaluate the role of AtG3BP1 in MTI, Atg3bp1 mutant lines were challenged with P. syringae pv. tomato DC3000 $\mathrm{hrCC}^{-}$(Pst hrcC $)$that 
A

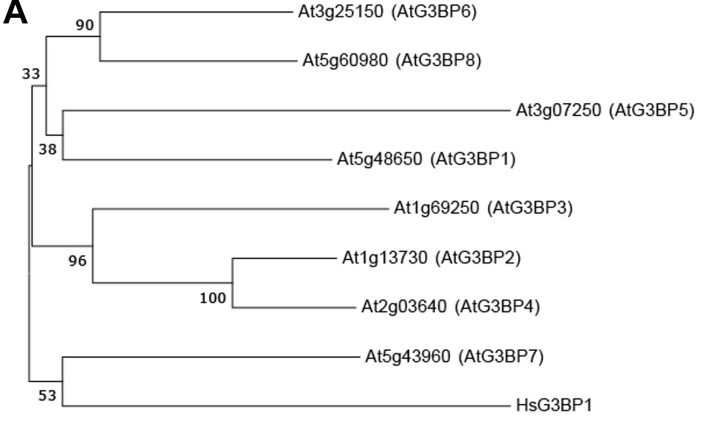

$\longdiv { 0 . 1 0 }$
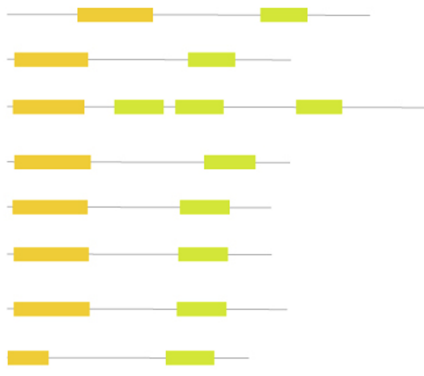

NTF2

RRM
Figure 1. AtG3BP1 is a homolog of the human HsG3BP1.

(A) Neighbor-joining phylogenetic tree of the AtG3BPs superfamily and HsG3BP1 and Schematic comparison of the domain architecture of the AtG3BPs superfamily. The following domains are indicated: nuclear transport factor 2 (NTF2) and RNA recognition motif (RRM). The optimal tree with the sum of branch length $=5.30337989$ is shown. The percentage of replicate trees in which the associated taxa clustered together in the bootstrap test (500 replicates) is shown next to the branches (Felsenstein, 1985). The tree is drawn to scale, with branch lengths in the same units as those of the evolutionary distances used to infer the phylogenetic tree. Evolutionary analyses were conducted in MEGA7 (Kumar et al, 2016). The evolutionary distances were computed using the Poisson correction method and are in the units of the number of amino acid substitutions per site. The analysis involved nine amino acid sequences. All positions containing gaps and missing data were eliminated. (B) AtG3BP1 protein structure showing the relative position of NTF2 and RRM domains. (C) The intron/exon structure of AT5G48650 and T-DNA insertional mutation sites in Atg3bp1-1 and Atg3bp1-2 mutant lines. Filled box represent exons, and AA155 and AA156 denote the positions of the primers used in the gene expression analysis. (D) Atg3bp1-1 and Atg3bp1-2 are loss-offunction mutants: expression levels of Atg3bp1-1 and Atg 3bp1-2 by qPCR relatively to Col-0 WT (set at 1) with primers spanning the third exon (AA155 and AA156). UBQ10 and actin expression levels were used for normalization. (E) Expression levels of AtG3BP1 OE2 and OE3 by GPCR relatively to Col-0 WT (set at 1) with primers spanning the $3^{\text {rd }}$ exon (AA155 and AA156). UBQ10 and actin expression levels were used for normalization. (F). Morphological phenotype of WT (Col.0), Atg3bp1-1, and Atg3bp1-2 mutants, and 35S::AtG3BP1-GFP (OE2) and 35S::AtG3BP1-GFP (OE3) transgenic lines. 4-week-old jiffy peat pellets-grown plants are shown.

\section{$\mathbf{F}$}

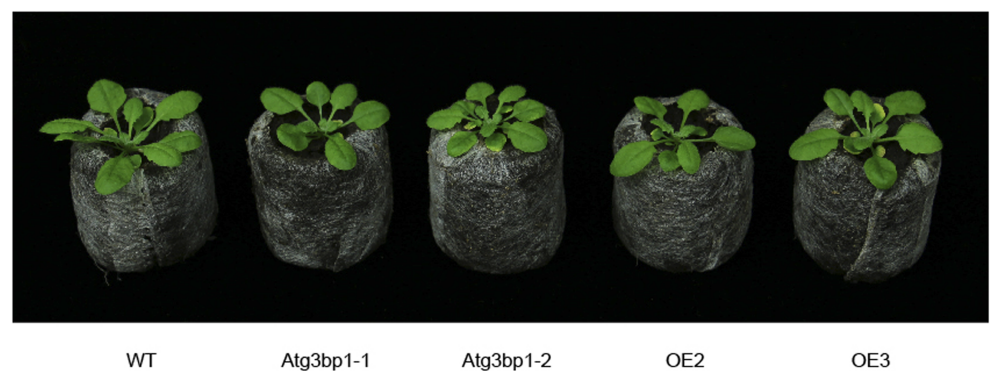

lacks the type III secretion system. Arabidopsis Atg3bp1-1 and Atg3bp1-2 mutants had reduced bacterial titers $3 \mathrm{hpi}$ and $72 \mathrm{hpi}$ compared with WT plants (Fig 3A). Conversely, the AtG3BP1 overexpressor lines OE2 and OE3 harbored higher bacterial titers $72 \mathrm{hpi}$ compared with WT plants (Fig 3B). Taken together, these data suggest that AtG3BP1 negatively regulates disease resistance to Pst $h r c C^{-}$. In addition, the difference in bacterial titers in the Atg3bp1-1 and Atg3bp1-2 mutants after 3 hpi suggests that AtG3BP1 regulates the entry of Pst hrcC into the plant.

Because Arabidopsis resistance to Pst $\mathrm{hrCC}^{-}$was increased in both Atg3bp1 mutant alleles Atg3bp1-1 and Atg3bp1-2 (Fig 3A), we looked for cellular responses involved in MTI that were deregulated.
We first evaluated an early MTI response, the production of ROS (Kadota et al, 2014) in both alleles and observed a stronger ROS burst in Atg3bp1 mutant lines compared with WT plants after treatment with flg22 (Fig 3C).

Because MTI has been reported to be associated with transcriptional reprogramming (Navarro et al, 2004; Zipfel et al, 2004), we investigated the role of AtG3BP1 in MAMP-triggered transcriptional responses. Quantitative RT-PCR (qRT-PCR) analysis showed that the classical MTI markers (Yang \& Klessig, 1996; Asai et al, 2002) FRK1, PR1, and WRKY29 were significantly more induced in untreated Atg3bp1-1 when compared with WT plants. Besides, flg22 treatment further induces the expression of FRK1 and PR1 in the mutants 
A

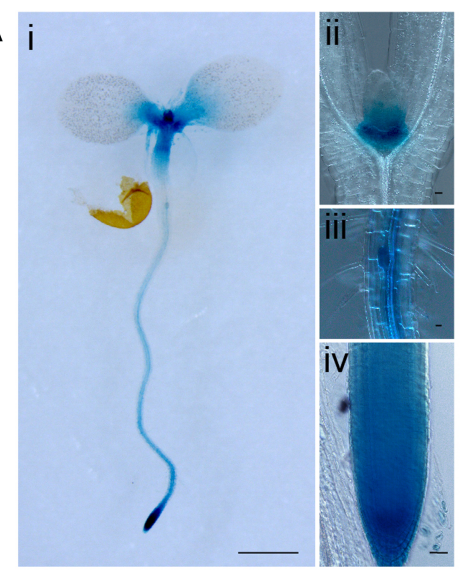

C
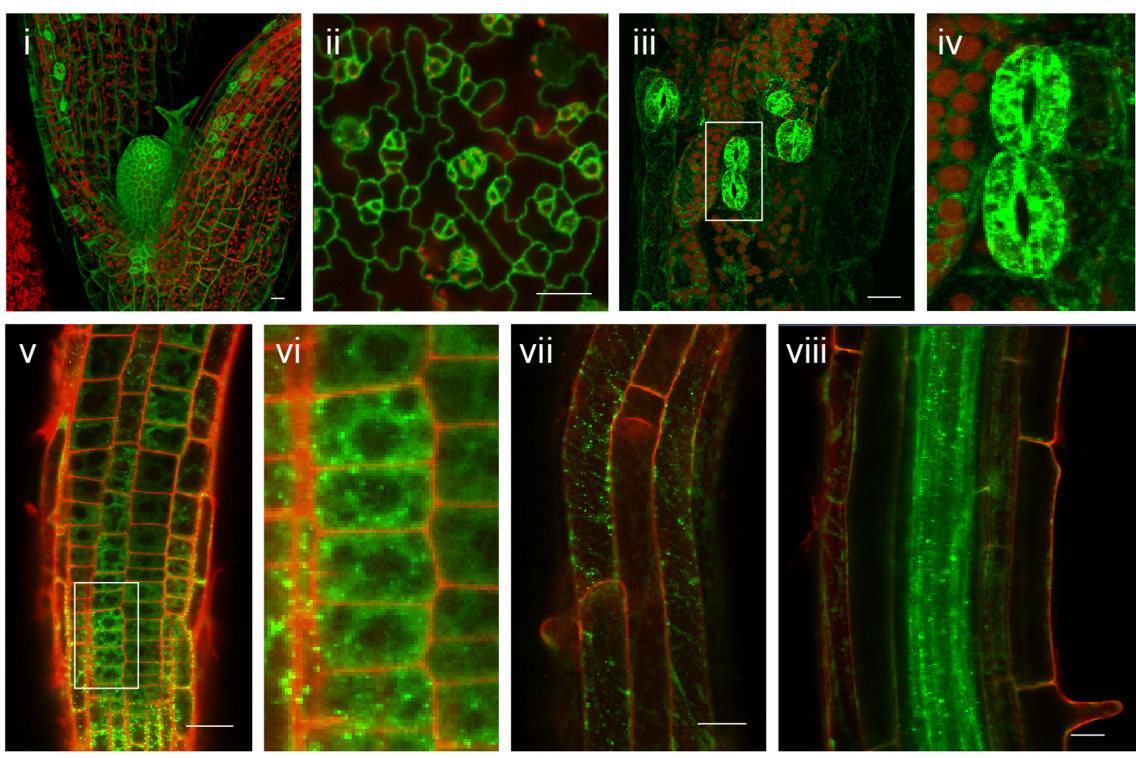

D
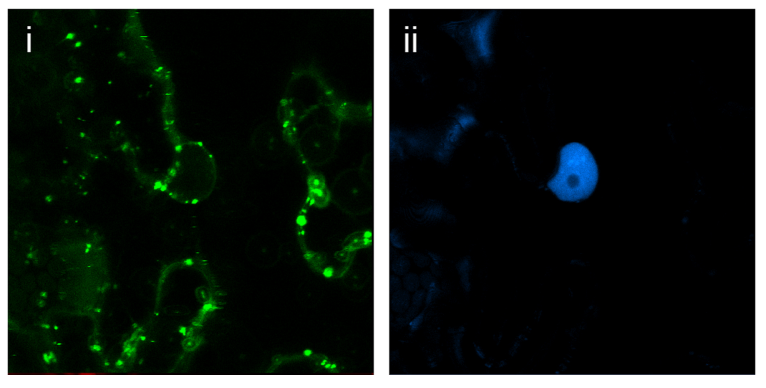

Figure 2. AtG3BP1 is expressed and localized to different cellular compartments.

(A) Expression patterns for the GUS reporter gene under the control of the AtG3BP1 promoter in transgenic Arabidopsis seedlings. Histochemical GUS staining at the seedling stage. (i) 8-d-old seedling stained for $2 \mathrm{~h}$. (ii) Shoot apical meristem of 14 -d-old seedling stained for $12 \mathrm{~h}$. (iii) Lateral root of 14-d-old seedling stained for 8 h. (iv) Root apical meristem of 12-d-old seedlings stained for $2 \mathrm{~h}$. Scale bar: (i) $=1,000 \mu \mathrm{m}$, (ii-iv) $=20 \mu \mathrm{m}$. (B) Immunoblot of WT, OE2 plants expressing 35S:: AtG3BP1-GFP probed with anti-GFP antibody (upper panel) and protein loading control with Ponceau S staining (lower panel). (C) AtG3BP1 expression and protein localization in Arabidopsis roots ( $8 \mathrm{~d}$ ) and leaves $(12 \mathrm{~d})$. Stable transgenic plants overexpressing AtG3BP1-GFP (OE2) reveal that AtG3BP1 is mainly localized to various cytoplasmic compartments. Confocal laser scanning microscopy images of GFP fluorescence (green) and propidium iodide (PI) fluorescence (red) of Arabidopsis seedlings in the (i) shoot apical meristem, (ii) leaf epidermis, and (iii) hypocotyl. Panel (iv) is the enlargement of boxed area in (iii), showing details of stomata localization. (v) Root apical meristem-epidermal layer. Panel (vi) is the enlargement of boxed area in (v), showing details of localization of AtG3BP1 to cytoplasmic compartments and structures. The protein is excluded from the nucleus. (vii, viii) Root differentiation zone. Scale bar: $20 \mu \mathrm{m}$. (D) Subcellular localization of transiently expressed 35S::AtG3BP1-GFP and pUBi::Serrate-CFP in $N$. benthamiana cells. The indicated combinations of fluorescently tagged proteins were transiently expressed in 4-week-old $N$. benthamiana leaf epidermal cells. The localization was visualized $72 \mathrm{~h}$ after infiltration by laser scanning confocal microscopy. GFP fluorescence is in green and CFP fluorescence is in blue. Scale bars $=20 \mu \mathrm{m}$. compared with WT plants. Although the expression of WRKY29 and MYB51 was higher in the mutants compared with the WT after flg22 treatment, this was statistically not significant (Fig 3D).

In plants, ROS act as signaling molecules and have been shown to play a role in multiple stress responses (Dalton et al, 1999). ROS can also induce a variety of auto-oxidative chain reactions leading to signaling events and ultimately to the destruction of organelles and macromolecules (Mittler et al, 2004; O'Brien et al, 2012). To further clarify the role of AtG3BP1 in stress responses, WT plants and Atg3bp1 mutants were specifically stained for superoxide radicals using the nitroblue tetrazolium (NBT) and for hydrogen peroxide using 3,3'-DAB. Polymerization of DAB and NBT can be detected as a brown precipitate in the presence of hydrogen peroxide and as a blue precipitate in the presence of superoxide, respectively. Compared with WT plants, a strong DAB and NBT stain appeared in Atg3bp1-1 and Atg3bp1-2 mutants (Fig 3E and F). 

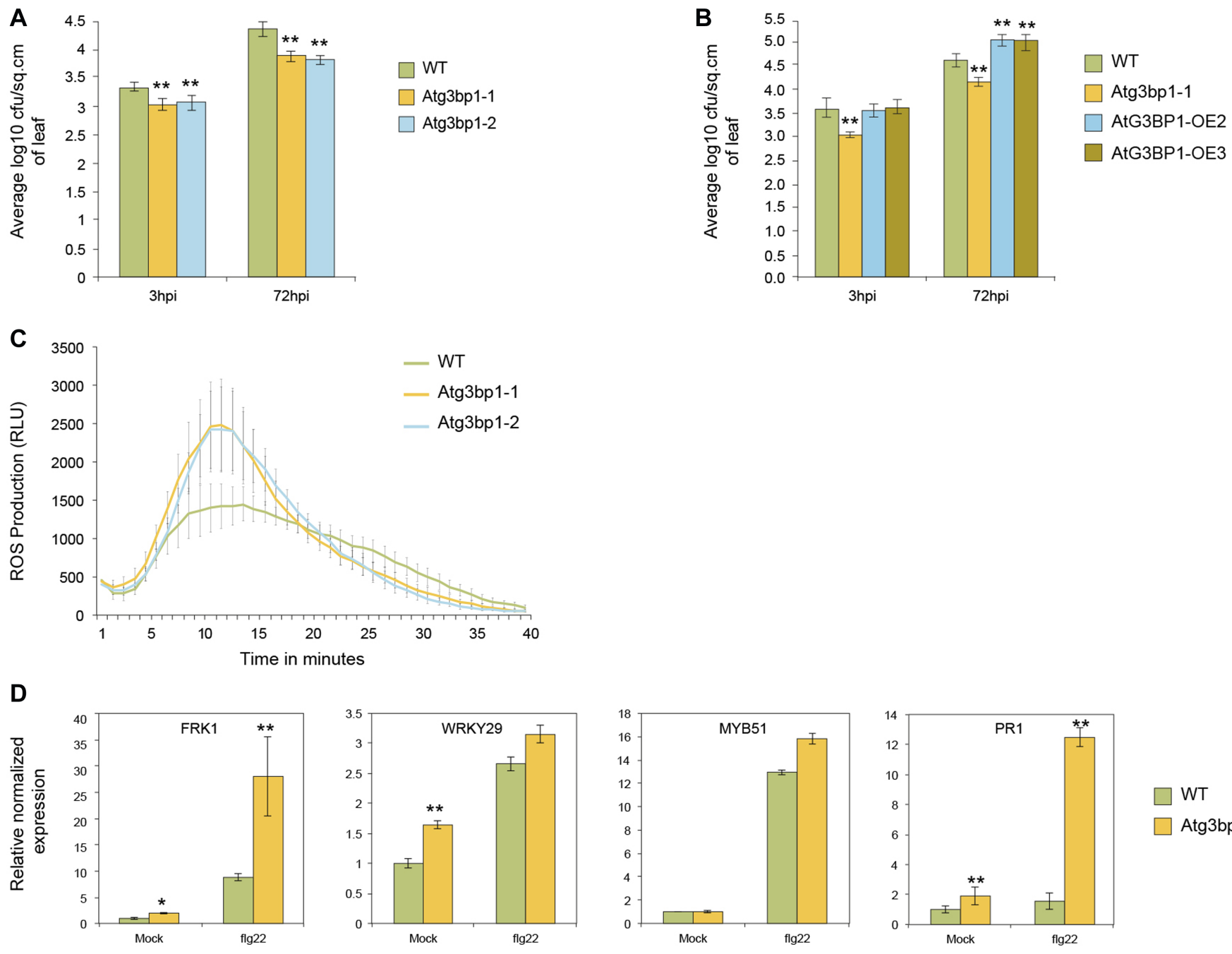

WT

Atg3bp1-1

E

WT Atg3bp1-1 Atg3bp1-2

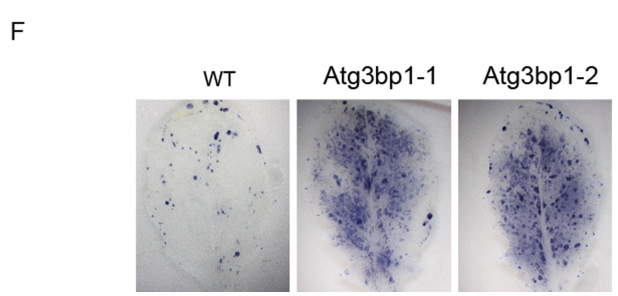

Figure 3. The Atg3bp1 mutants display enhanced disease resistance and alter classical MTI responses.

(A) Bacterial titers evaluated 3 and $72 \mathrm{~h}$ after spray inoculation with $1 \times 10^{8} \mathrm{CFU}_{\mathrm{ml}}{ }^{-1}$ Pst hrcC $\mathrm{C}^{-}$in WT and two Atg3bp1 mutant lines (Atg3bp1-1 and Atg3bp1-2). Values represent SEM from three independent experiments. Asterisks indicate significant differences from the WT as determined by Mann-Whitney $U$ two-tailed test $(P \leq 0.01)$. (B) Bacterial titers evaluated 3 and $72 \mathrm{~h}$ after spray inoculation with $1 \times 10^{8} \mathrm{CFU} \cdot \mathrm{ml}^{-1} \mathrm{Pst}$ hrcC $\mathrm{C}^{-}$in WT, Atg3bp1-1, and two AtG3BP1 overexpressing lines (OE2 and OE3). Values represent SEM from three independent experiments. Asterisks indicate significant differences from the WT as determined by Mann-Whitney $U$ two-tailed test $(P \leq 0.01)$. (C) flg22-induced ROS burst in the WT and Atg3bp1 mutant lines. Leaf discs from 4-week-old plants were treated with $1 \mu \mathrm{M}$ flg22 over 40 min. The data were shown as means from 12 leaf discs. (D) flg22-induced marker gene expression in the WT and Atg3bp1-1 plants. 14-d-old seedlings were treated with $1 \mu \mathrm{M}$ flg22 for $1 \mathrm{~h}$. Transcripts levels were determined by qRT-PCR relatively to Col-0 WT (set at 1). UBQ10 and actin expression levels were used for normalization. (E, F) Detection of hydrogen peroxide accumulation by DAB staining (F) and of superoxide anion accumulation by NBT staining (G) in WT and Atg3bp1 mutants.

\section{AtG3BP1 is a negative regulator of stomatal immunity}

Plants restrict the invasion of bacteria after sensing the MAMPs through stomatal closure (Melotto et al, 2006; Zeng et al, 2010). We then hypothesized that the lower bacterial titers that we observed
3 hpi in Atg3bp1 mutant lines compared with WT after spray inoculation (Fig 3A) might result from the prevention of bacterial entry into the leaves via the stomata. To test this hypothesis, we examined the stomatal aperture in Atg3bp1-1 mutants. Stomata of Atg3bp1-1 were more closed compared with those of WT plants 
(Fig 4A), suggesting that diminished levels of AtG3BP1 caused the stomatal response phenotype observed in Atg3bp1-1 and Atg3bp1-2.

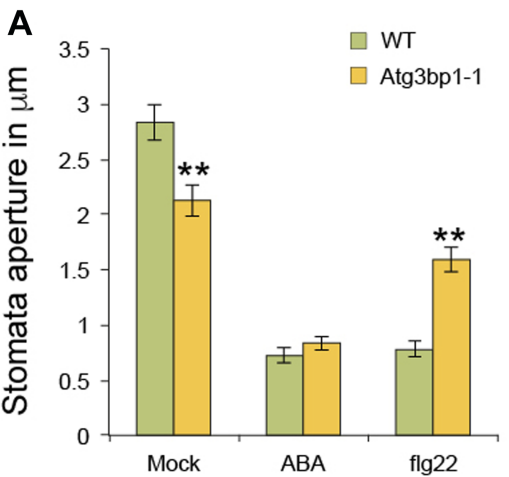

\section{C}

B
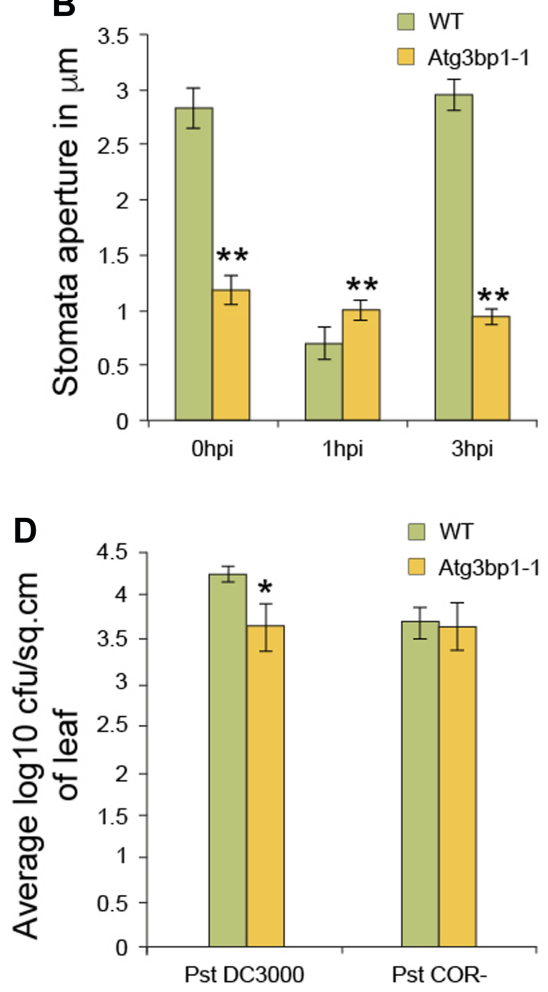

$\mathbf{F}$

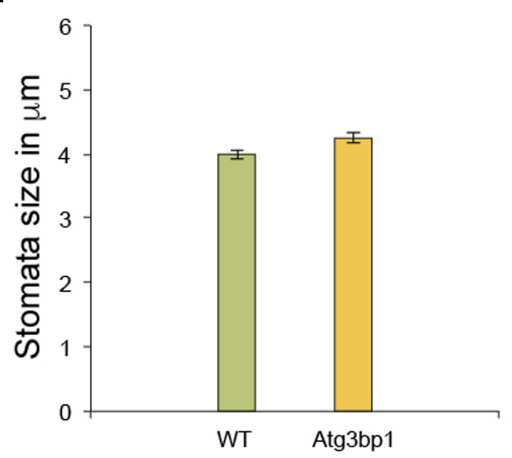

H

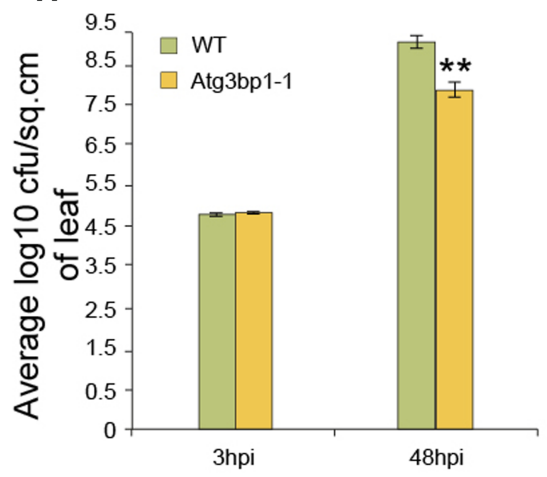

We then treated Atg3bp1-1 mutant lines with flg22 or abscisic acid (ABA), both of which are known to promote stomatal closure. After $1 \mathrm{~h}$ of ABA treatment, the stomata in both WT and Atg3bp1-1
E

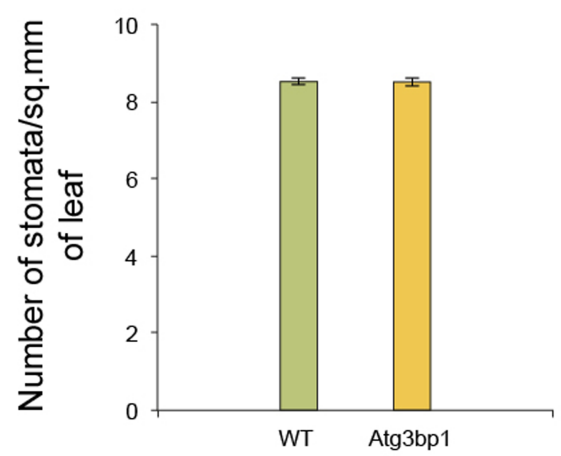

G

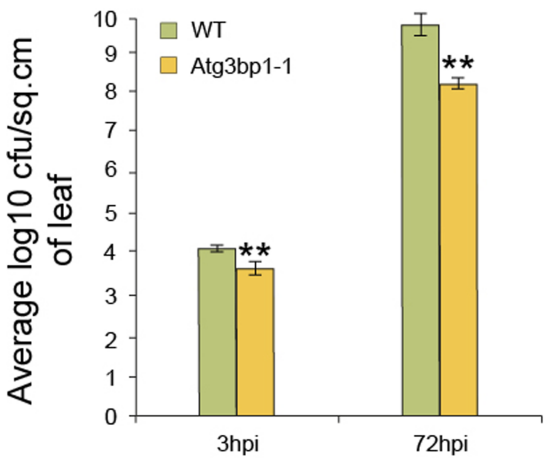

Figure 4. AtG3BP1 is associated with stomatal immunity.

(A) Stomata closure upon ABA and flg22 treatment Epidermal peels of 5-week-old WT and Atg3bp1-1 plants were floated in stomata buffer with either $20 \mu \mathrm{M}$ ABA or $1 \mu \mathrm{M}$ flg22. Stomatal apertures were evaluated after $1 \mathrm{~h}$. Values represent SEM from three independent experiments. Asterisks indicated significant differences from the WT plants determined by a Mann-Whitney $U$ two-tailed test $(P \leq 0.01)$. (B, C) Stomata closure upon Pst or Pst COR ${ }^{-}$inoculation. Epidermal peels of 5-weekold WT and Atg3bp1-1 plants were floated in a suspension of $1 \times 10^{8} \mathrm{CFU} \cdot \mathrm{ml}^{-1}$ Pst or Pst COR ${ }^{-}$in stomata buffer. Stomatal apertures were evaluated at the indicated time points. Values represent SEM from three independent experiments. Asterisks indicate significant differences from the WT plants determined by a Mann-Whitney $U$ two-tailed test $(P \leq 0.01)$.

(D) Bacterial titers evaluated $3 \mathrm{~h}$ after spray inoculation with $1 \times 10^{8} \mathrm{CFU} \cdot \mathrm{ml}^{-1}$ Pst or Pst COR ${ }^{-}$in WT and Atg3bp 1-1 plants. Values represent SEM from three independent experiments. Asterisks indicate significant differences from the WT plants as determined by a Mann-Whitney $U$ two-tailed test $(P \leq 0.05)$. (E) Stomata density in leaf abaxial surfaces of WT and Atg3bp1-1 (D, stomata per $\mathrm{mm}^{2}$ ). (F). Average stomatal size in leaf abaxial surfaces of WT and Atg3bp1-1. Mean pore depth ( $\mathrm{mm}$ ), which is assumed to be equivalent to guard cell width. (G) Bacterial titers evaluated 3 and $72 \mathrm{~h}$ after spray inoculation with $1 \times 10^{8} \mathrm{CFU} \cdot \mathrm{ml}^{-1}$ Pst in WT and Atg3bp11. Values represent SEM from three independent experiments. Asterisks indicate significant differences from the WT plants as determined by a Mann-Whitney $U$ two-tailed test $(P \leq 0.01)$. (H) Bacterial titers evaluated 3 and $48 \mathrm{~h}$ after infiltration inoculation with $1 \times 10^{8}$ $\mathrm{CFU} \cdot \mathrm{ml}^{-1}$ Pst in WT and Atg3bp1-1 plants. Values represent SEM from three independent experiments. Asterisks indicate significant differences from the WT plants as determined by a Mann-Whitney $U$ two-tailed test $(P \leq 0.01)$. 
plants closed to the same extent, whereas the stomatal closure in Atg3bp1-1 mutant lines was compromised in response to MAMP treatment (Fig 4A). This observation shows that although the ABAdependent stomatal closure is not affected in the Atg3bp1-1 mutants, the flg22-dependent pathway is compromised, confirming a role for AtG3BP1 in the stomatal immune pathway.

Arabidopsis plants close stomata when they sense Pst within $1 \mathrm{hpi}$. However, Pst and other virulent bacteria induce the stomata to reopen via secretion of the chemical effector COR. Thus, WT Arabidopsis are found to be resistant to COR-deficient Pst mutants of Pst COR (Melotto et al, 2006; Zeng et al, 2010). To test the role of AtG3BP1 in stomatal reopening, leaf pieces were incubated with Pst or Pst COR bacteria. Stomatal apertures in leaf epidermal peels of WT and Atg3bp1-1 plants were subsequently assessed after 1 and 3 h. In WT plants, the stomata rapidly closed after treatment with Pst and reopened to normal levels after 3 hpi (Fig 4B). However, when compared with WT plants, Atg3bp1-1 mutants were compromised in stomatal closure in nontreated controls at 0 hpi and $1 \mathrm{~h}$ after treatment (Fig 4B and C at 0 and $1 \mathrm{hpi}$, respectively). Importantly, Atg3bp1-1 plants were completely insensitive to Pst-mediated stomatal reopening at $3 \mathrm{hpi}$ (Fig 4C, $3 \mathrm{hpi}$ ). These data indicate that Atg3bp1-1 mutants counteract CORdependent reopening of stomata and that resistance to Pst $\mathrm{hrCC}^{-}$in Atg3bp1 mutant lines may, therefore, be due to the stomatal closure response.

To further evaluate the role of AtG3BP1 in stomatal immunity, WT plants and Atg3bp1-1 mutant lines were challenged with Pst and Pst COR by spray inoculation. Bacterial titers were evaluated at $3 \mathrm{hpi}$. Bacterial titers were significantly reduced in Atg3bp1-1 compared with WT plants when challenged with Pst (Fig 4D). However, no significant differences in the bacterial titers of Atg3bp1-1 and WT were found when challenged with Pst $\mathrm{COR}^{-}$(Fig 4D). We also measured the stomatal density and size in Atg3bp1-1 for developmental defects. However, Atg3bp1-1 mutant and WT showed similar stomatal density and size (Fig 4E and $\mathrm{F}$ ), indicating that the reduced bacterial titers in Atg3bp1-1 were not the result of smaller stomatal size or density but due to differences in stomatal behavior.

We further tested the disease phenotype of Atg3bp1 mutant lines with the virulent bacterial pathogen Pst. Atg3bp1-1 developed less disease symptoms and had lower bacterial titers 3 and 72 hpi than WT controls when challenged with Pst by spray inoculation (Fig 4G). Next, we investigated whether AtG3BP1 is critical for Arabidopsis resistance even when the stomatal barrier was breached by infiltration inoculation of Pst, which bypasses the first barrier of defense, namely, stomatal closure that restricts the passage of bacteria into the plant (Melotto et al, 2006; Zeng et al, 2010). However, Atg3bp1-1 showed an enhanced resistance phenotype when challenged with Pst by infiltration inoculation (Fig $4 \mathrm{H}$ ), indicating that AtG3BP1 also negatively regulates postinvasive disease resistance in mesophyll cells.

\section{Global transcriptomic profile shows that AtG3BP1 negatively regulates defense gene expression}

To identify the AtG3BP1-dependent genes, we performed whole transcriptome analysis (RNA-seq) with 14-d-old seedlings of
WT and Atg3bp1-1 genotypes treated for $24 \mathrm{~h}$ with or without $1 \times 10^{8}$ CFU. $\mathrm{ml}^{-1}$ Pst hrcC $^{-}$by spray inoculation. Samples from three independent biological experiments were collected for RNA extraction and RNA-seq was carried out on Illumina HiSeq (Illumina Inc.). The data discussed in this publication have been deposited in NCBI's Gene Expression Omnibus (GEO) and are accessible through GEO Series accession number GSE107786. Differentially expressed genes (DEGs) were based on at least a twofold change in gene expression and the false discovery rate (FDR) value $(P \leq 0.05)$ as described in the Bioinformatics part of the Materials and Methods section (Fig S3A). All the down- or up-regulated genes were overlapped to obtain the unique down- or up-regulated genes in mock-mock and mock-Pst hrcC $\mathrm{C}^{-}$treated conditions (Fig 5A).

Performing hierarchical clustering by combining all the 1937 unique genes that are differentially expressed (Fig 5A) revealed several clusters as depicted in Fig 5B. Focusing on three specific clusters of interest, cluster IV comprised 160 DEGs that were highly up-regulated in Atg3bp1-1 upon Pst hrcC treatment but not in WT (Fig 5C). Enrichment analysis of Gene Ontology categories of cluster IV revealed genes associated with response to stress, biotic stimulus, and defense (Fig 5C). Therefore, Atg3bp1 mutants respond more strongly to microbial challenge than WT. A constitutive defense response in Atg3bp1-1 was observed for cluster VI, with 261 DEGs, which were highly up-regulated already in the absence of Pst hrcC ${ }^{-}$treatment (Fig 5C). Another large set of genes, cluster VII with 597 DEGs, all related to ROS, hydrogen peroxide metabolism, oxidationreduction process, peroxidase activities, and oxidoreductase activities, was also found to be constitutively up-regulated in untreated Atg3bp1-1 plants (Fig 5C).

The expression of several immunity-related genes in Atg3bp1 mutants, including WAK1 (AT1G21250), WRKY40 (AT1G80840), WRKY8 (AT5G46350), and AZI1 (AT4G12470), and defensin-like protein (AT3G59930) was confirmed by qRT-PCR analysis (Fig S3B). Taken together, the global gene expression data suggest that AtG3BP1 plays a negative role in a large subset of immunity-related genes.

\section{AtG3BP1 is a key negative regulator of genes involved in SA biosynthesis and signaling}

The transcriptome data indicated that a large set of immunityrelated genes are either constitutively or more highly induced in Atg3bp1 mutants upon microbial challenge. Among these genes, a strong enrichment was observed for SA-related genes (Fig S3C and D). For this purpose, we evaluated the expression levels of several known SA-mediated defense genes in Atg3bp1-1 and WT seedlings by qRT-PCR. We found that the basal transcript levels of the CaM-binding protein $60 \mathrm{~g}$ (CBP60g) and SAR deficient-1 (SARD1) were higher in Atg3bp1-1 than those in WT. CBP60g and SARD1 are members of the CaM-binding protein family that regulate the expression of the SA biosynthetic gene isochorismate synthase 1 (ICS1) (Zhang et al, 2010; Wang et al, 2011). The expression of ICS1 was also significantly higher in Atg3bp1-1 compared with WT (Fig 6A). For SA signaling-related genes, the expression levels of NIM1 interacting 1 (NIMIN1), transcription factor WRKY38, and PR2 were tested and found to be higher in Atg3bp1-1 compared with WT (Fig 6B). Expression of some of the SA marker genes PAD4, EDS1, and EDS5 were also elevated in Atg3bp1-1 compared to WT (Fig 6C). The 
A

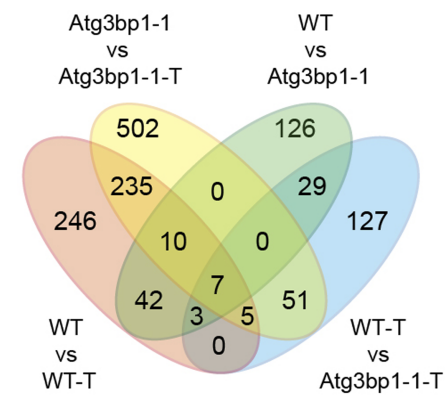

Down regulated genes

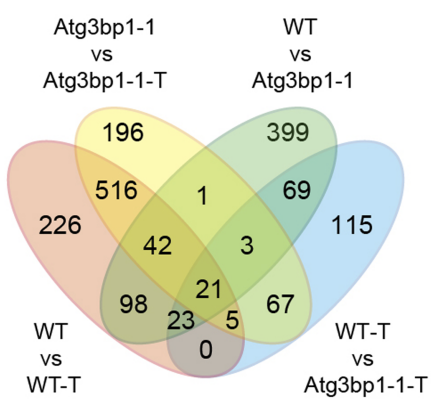

Upregulated genes

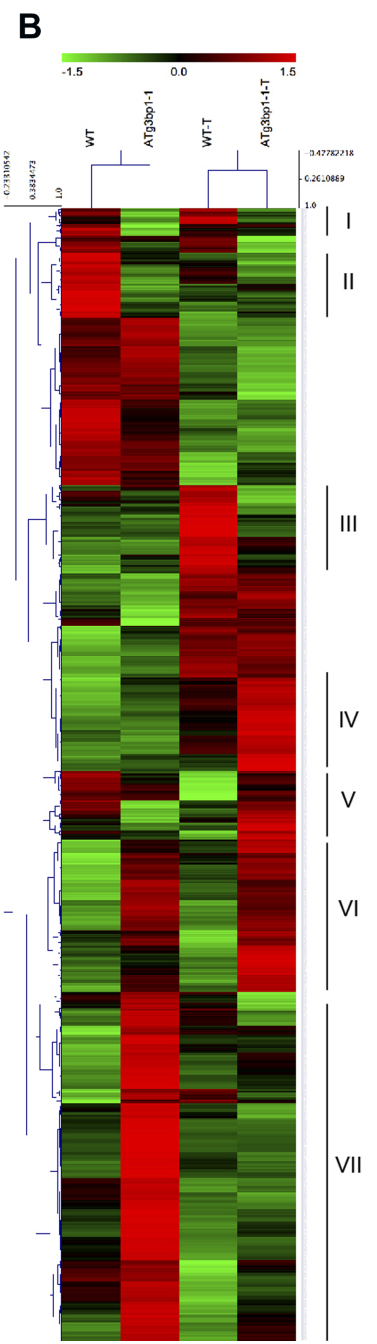

C
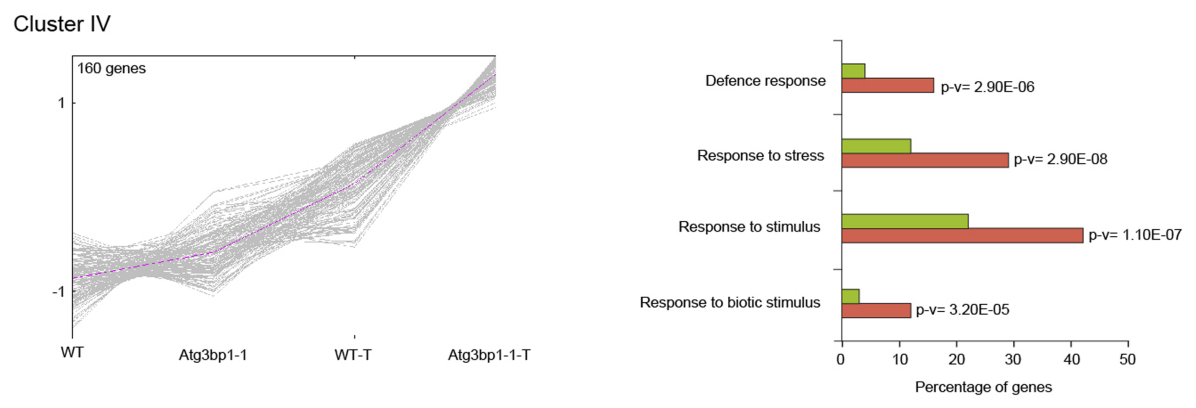

Background

Input list

Cluster VI
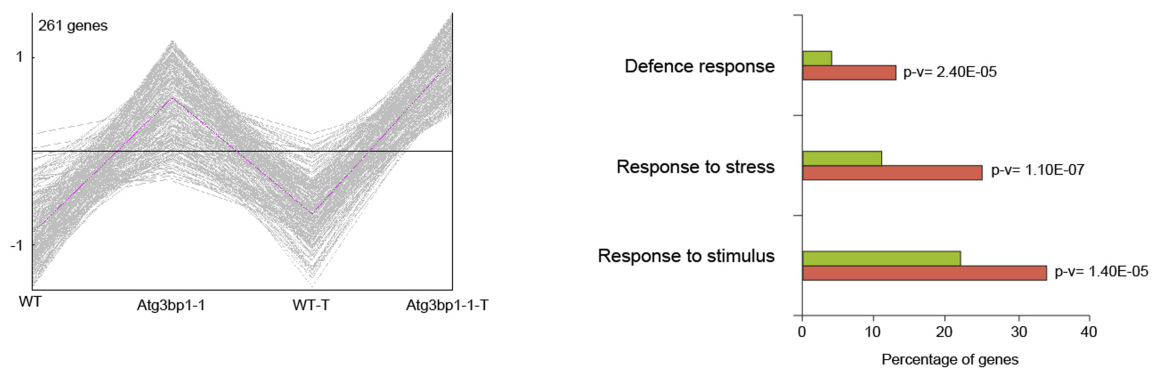

Cluster VII
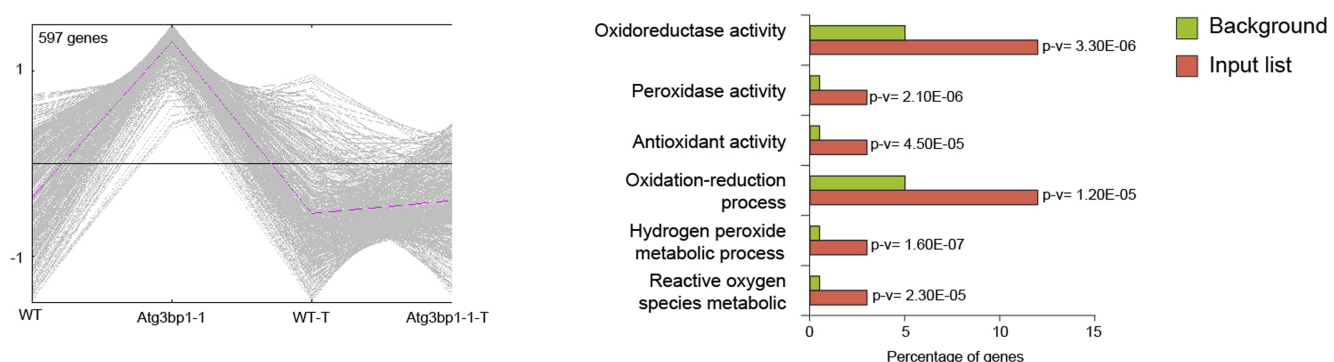

Figure 5. Global transcriptomic profile shows that AtG3BP1 is associated with defense responses.

(A) Venn diagrams showing the number of the unique differentially expressed genes. The numbers in the Venn diagrams were obtained by overlapping the unique downor up-regulated genes in mock-mock and mock-treated conditions (WT versus WT-treated, Atg3bp1-1 versus Atg3bp1-1-treated, WT versus Atg3bp1-1, and WT-treated versus Atg3bp1-1-treated). (B) Heat map of AtG3BP1-induced genes with and without Pst hrcC ${ }^{-}$treatment in WT and Atg3bp1-1 plants. The original fragments per kilobases million values were subjected to data adjustment by normalizing genes or rows and hierarchical clustering was generated with the average linkage method using MeV4.0. Red color indicates up-regulation and green indicates down-regulation. Clusters are defined in the text. (c) Expression profiles and enrichment of genes with GO terms for interesting clusters of AtG3BP1-induced genes with and without Pst hrcC ${ }^{-}$treatment in WT and Atg3bp1-1 plants. Right panel: for each interesting cluster, enrichment in genes with GO terms related to immune response. The fold enrichment was calculated based on the frequency of genes annotated to the term compared with their frequency in the genome. 

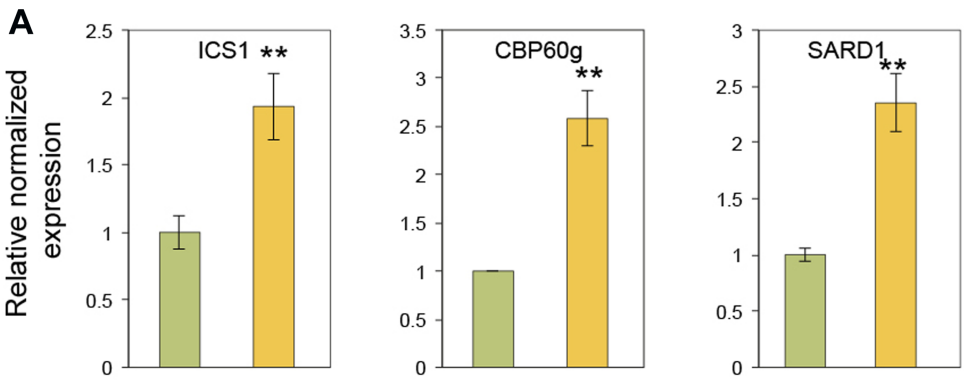

WT

Atg3bp1-1
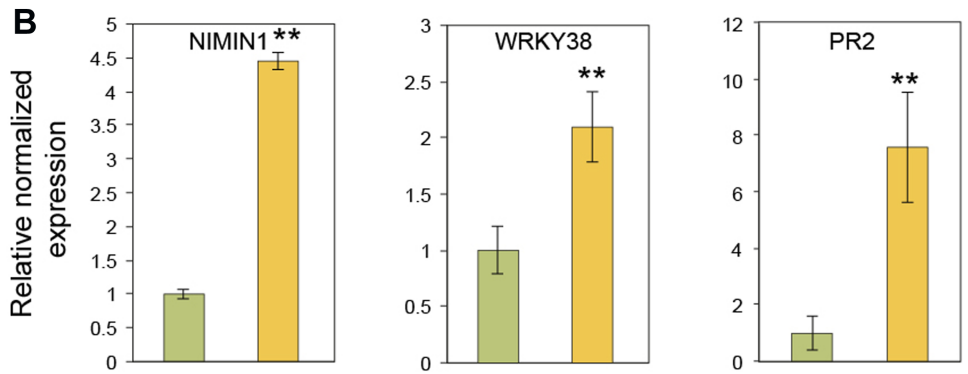

WT

Atg3bp1-1
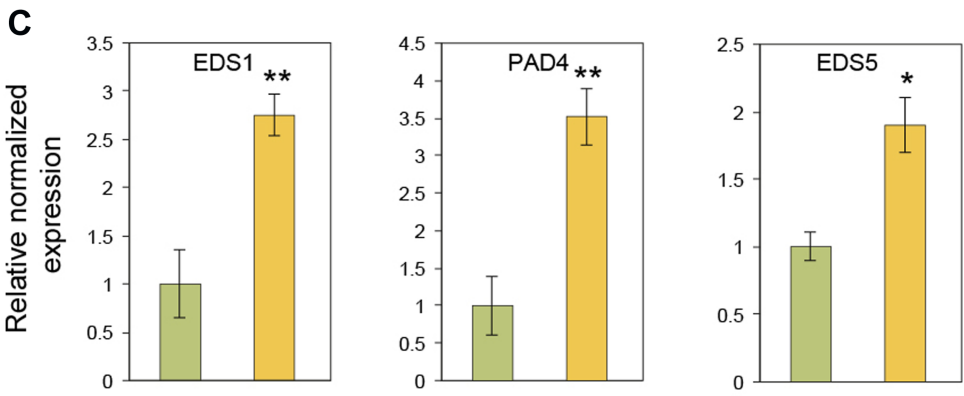

WT

Atg3bp1-1
Figure 6. Endogenous salicylic acid (SA) triggers stomatal closure and immune defense in Atg3bp1 mutants.

(A) Expression of SA-biosynthesis-related genes in the WT and Atg3bp1-1 14-d-old seedlings. Transcript levels were determined by qRT-PCR relatively to Col-0 WT (set at 1). UBQ10 and actin expression levels were used for normalization. (B) Expression of SA-signaling-related genes in the WT and Atg3bp1-1 14-d-old seedlings.

Transcript levels were determined by qRT-PCR relatively to Col-0 WT (set at 1). UBQ10 and actin expression levels were used for normalization. (C) Expression of SAaccumulation-related genes in the WT and Atg3bp1-1 14-d-old seedlings. Transcript levels were determined by qRT-PCR relatively to Col-0 WT (set at 1). UBQ10 and actin expression levels were used for normalization. (D) Endogenous SA levels in 14-d-old seedlings of WT and Atg3bp1-1 mutant plants. Values represent SEM from three independent experiments. (E) Stomatal aperture in epidermal peels of 5-week-old WT and Atg3bp1-1 plants were floated in stomata buffer and treated $1 \mathrm{~h}$ with $100 \mu \mathrm{M}$ SA followed by inoculation with either Pst or Pst COR-. Stomatal apertures were evaluated after 3 h. Values represent SEM from three independent experiments. Asterisks indicate significant differences from WT plants determined by a Mann-Whitney $U$ twotailed test $(P \leq 0.01)$.
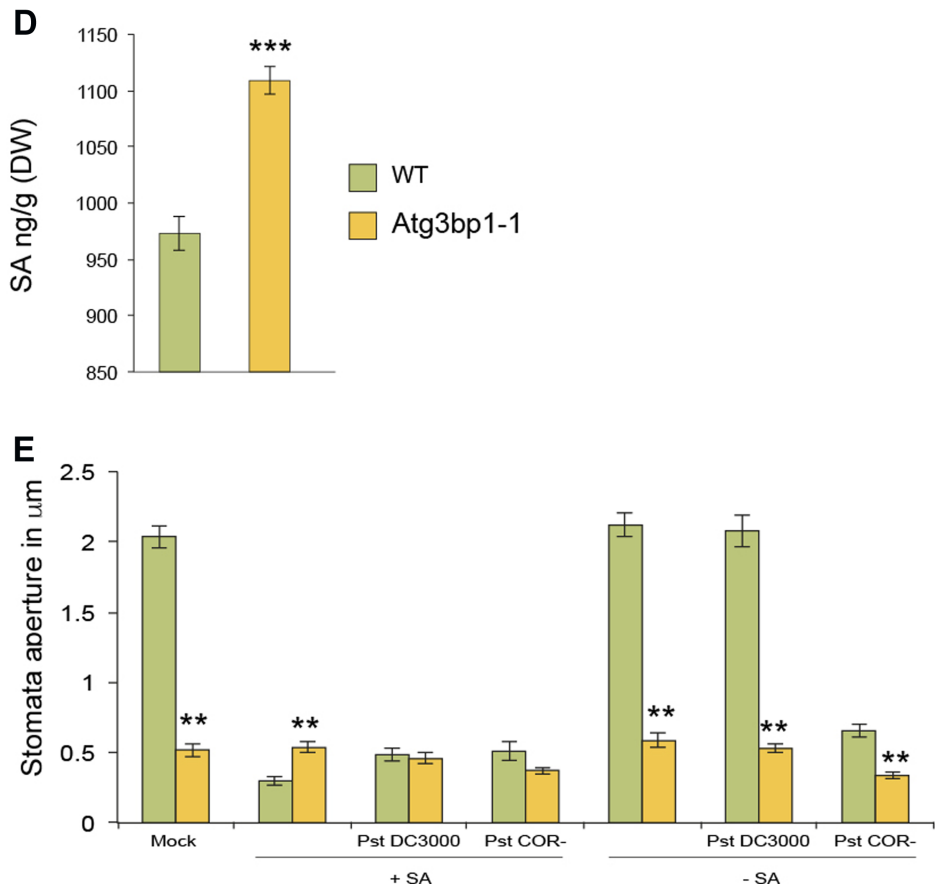

WT

Atg3bp1-1 
transcription factors WRKY40 and AZI1 (azelaic acid induced-1) are often used as markers for SA-dependent host defense responses (Lamb et al, 1989; Baker et al, 1997). WRKY40 and AZI1 were also upregulated in Atg3bp1-1 compared with WT controls (Fig S3B). Overall, the hyperinduction of SA defense-related genes correlates with the resistance to Pst observed in the Atg3bp1 mutants.

\section{AtG3BP1 regulates stomatal immunity via SA signaling}

We showed that Arabidopsis G3BP1 is critical for stomatal immunity and that AtGBBP1 is a negative regulator of SA biosynthesis and signaling gene expression. Since $S A$ is required for innate immunity-mediated stomatal closure (Zeng et al, 2011b), we thus quantified endogenous SA levels in Atg3bp1 mutant lines and found significant increase in SA levels in Atg3bp1-1 mutants compared with WT seedlings (Fig 6D).

Because an increase in endogenous SA can trigger stomatal closure, we hypothesized that an increase in the expression of SArelated genes might trigger stomatal closure and in turn lead to immune resistance in Atg3bp1 mutants. For this purpose, stomatal apertures were assessed in WT and Atg3bp1-1 after a 1-h pretreatment of SA followed by inoculation with either Pst or Pst COR ${ }^{-}$. The inoculation with PSt did not reopen the stomata in WT when pretreated with SA. But in WT leaves that had not been treated with $\mathrm{SA}$, stomata completely reopened to normal sizes after $3 \mathrm{~h}$ of inoculation with Pst because of COR produced by the pathogen. In WT leaves pretreated with SA followed by inoculation with PSt COR', the stomata remained closed because of the lack of COR. In contrast, the stomata in Atg3bp1-1 leaves remained closed and the treatment with either Pst or Pst COR- had no effect on the stomata (Fig 6E). Collectively, these results confirm that AtG3BP1 functions in SAmediated stomatal closure and reopening in response to bacterial pathogens.

\section{Discussion}

In this study, we report the identification of the Arabidopsis G3BP1 as an important player in SA-dependent stomatal immunity. AtG3BP1 is a member of a family of eight proteins in Arabidopsis that are orthologs to the human G3BP gene family. Mammalian G3BP1 is an evolutionarily highly conserved RBP that was identified through its interaction with a Ras-GTPase-activating protein (Tourriere et al, 2003). G3BPs have been mainly studied in mammals where they are involved in many processes, such as the formation of SGs and the involvement in virus resistance (Tourriere et al, 2003; Katsafanas \& Moss, 2007; Panas et al, 2012). However, so far, no G3BP has been functionally characterized in plant stomatal and apoplastic immunity. Expression profiles of the different members of the family in Arabidopsis are subjected to changes according to developmental stages, tissue specificity, and environmental perturbations. From these patterns, the genes might perform similar biochemical functions in a tissue- and developmental-specific manner and respond to different conditions (Fig S4). AtG3BP7 (At5g43960), formerly known as AtG3BP-like protein, was identified as an Arabidopsis G3BP-like protein that localizes to plant SGs and plays a role in the Arabidopsis virus resistance. It has been shown that SG formation and function is conserved between mammalian and plant cells during stress. Moreover, plant viruses have the ability to bind to AtG3BP7, preventing the formation of SGs, as in mammals (Krapp et al, 2017).

The enhanced disease resistance to Pst observed in two independent Atg3bp1 loss-of-function mutant lines indicates a role for AtG3BP1 in plant innate immunity. Several lines of evidence also showed that AtG3BP1 is involved in MTI. Atg3bp1 mutants have reduced sensitivity to Pst $h_{r C C}$, a nonvirulent pathogen used to study MTI. Atg3bp1 mutants also showed enhanced ROS accumulation in response to flg22 and up-regulation of classical MTI marker genes and stomatal innate immunity.

Atg3bp1-1 mutants showed the activation of defense responses leading to constitutive stomatal closure. More importantly, the striking insensitivity to COR-dependent stomatal reopening in Atg3bp1 mutants explains the enhanced resistance phenotype of Atg3bp1 mutants to Pst by spray inoculation. Moreover, in spite of overcoming the stomatal barrier by infiltration of Pst, the Atg3bp1 mutants showed enhanced disease resistance. Taken together, these data indicate that Arabidopsis G3BP1 is a regulator of stomatal and apoplastic immunity.

The analysis of the SA-deficient nahG mutant and SAbiosynthetic sid2/eds16 mutant revealed that SA biosynthesis is required for MAMP-induced stomatal closure (Melotto et al, 2006; Zeng et al, 2010). Atg3bp1 mutant plants showed up-regulation of SA biosynthesis and signaling marker genes and accumulated SA. It was also shown that SA induces stomatal closure by peroxidasemediated extracellular ROS production (Mori et al, 2001; Khokon et al, 2011). Consistent with a role of AtG3BP1 in SA-induced ROS production, Atg3bp1 mutants showed constitutive up-regulation of genes related to ROS production and enhanced DAB and NBT staining for hydrogen peroxide and superoxide, respectively. The SA-dependent pathway is also critical in mediating Pst resistance in the COR-insensitive coi-20 mutants (Kloek et al, 2001). Thus, SA signaling pathway is required for stomatal immunity and apoplastic defenses against Pst (Kloek et al, 2001; Melotto et al, 2006; Zeng et al, 2010, 2011a). Our data support the idea that Atg3bp1 immunity against Pst including stomatal defense is correlated with the activation of SA signaling.

Recently, COR was found to induce the NAC transcription factors (petunia NAM and Arabidopsis ATAF1, ATAF2, and CUC2) ANAC019, ANAC055, and ANAC07 to repress ICS1 and induce BSMT1 to inhibit SA accumulation, thus mediating reopening of the stomata to facilitate bacterial entry (Zheng et al, 2012). Interestingly, in Atg3bp1-1 mutants, two of these NAC TFs (ANAC019 and ANAC055) were upregulated in untreated plants (Fig S5) but nonetheless result in accumulation of SA, requiring another explanation for the insensitive phenotype to COR-induced stomatal reopening.

RBPS, especially those involved in response to stress, have been shown to regulate gene expression at different stages, from transcription to posttranslational levels (Mastrangelo et al, 2012). Posttranscriptional regulations are important processes in eukaryotes that enable them to adapt to changes in their environment. Human G3BP1 is located in SGs, which are sites of mRNA storage during stress responses that are involved in regulating mRNA stability and translatability (Anderson \& Kedersha, 2008). In 
humans, SGs are highly dynamic entities that recruit many signaling proteins in a transient manner until the cells adapt to stress or die. Hence, beyond their role as mRNA triage centers, SGs are proposed to constitute RNA-centric signaling hubs analogous to classical multiprotein signaling domains such as transmembrane receptor complexes which communicate a "state of emergency" (Kedersha et al, 2013). A good example for this as a signaling hub is the one shown by HsG3BP1 which functions in regulating double-stranded RNA-dependent protein kinase activation (Reineke et al, 2015). Given the highly conserved nature of eukaryotic G3BPs, it is tempting to speculate that plant G3BP1 might function in an analogous fashion during stress. This might also explain the fact that Atg3bp1 mutants showed enhanced ROS accumulation in response to flg22, which would suggest that AtG3BP1 functions upstream of ROS production. In this context, it should also be noted that ROS production does not only act as an antimicrobial agent in MTI, but also as a secondary messenger that triggers downstream defense responses, including stomatal closure and up-regulation of MTI marker genes (Melotto et al, 2006; Kadota et al, 2014; Yu et al, 2017).

Interestingly, whereas we identified eight G3BPs in Arabidopsis, the human genome only encodes for two HsG3BPs. Expression analysis of the Arabidopsis genes shows, however, that these AtG3BPs are expressed in a highly tissue- and developmentalspecific fashion (Fig S4), suggesting that the different genes might perform similar functions in different cellular contexts.

Collectively, this work reveals that plants and mammals possess a set of highly conserved G3BP proteins with a role in immunity and further investigations are warranted to unravel the roles of G3BPs in plants.

\section{Materials and Methods}

\section{Biological materials and growth conditions}

Arabidopsis ecotype Columbia-0 plants were used as WT in all experiments. Two T-DNA mutant lines Atg3bp1-1 (SAIL_1153_H01) and Atg3bp1-2 (SALK_027468) were obtained from the National Arabidopsis Stock Center (NASC) and genotyped by PCR amplification of insertion-specific or WT-specific fragments with primers listed in Table S1. Plants were grown on soil at $21^{\circ} \mathrm{C}$ with a $12-\mathrm{h}$ photoperiod or as seedlings on sterile one-half-strength Murashige and Skoog (MS) medium with a 16- or 8-h photoperiod for $14 \mathrm{~d}$.

Bacterial strains P. syringae pv. tomato Pst DC3000, Pst DC3000 hrcC, and Pst DC3000 COR were cultured at $28^{\circ} \mathrm{C}$ from glycerol stock on NYGA agar plate ( $5 \mathrm{~g} /$ liter bactopeptone, $3 \mathrm{~g} /$ liter yeast extract, $20 \mathrm{ml} /$ liter glycerol, and $15 \mathrm{~g} /$ liter agar) containing $50 \mathrm{mg} / \mathrm{ml}$ rifampicin.

\section{Cloning and generation of overexpressor plants}

All the details are described in the Materials and Methods section of the Supplemental Information.

\section{Various treatments, pathogen infection, and MTI assays}

MAMP and hormone treatments, bacterial pathogen infection assays, stomatal aperture assay, oxidative burst assay, superoxide radical and hydrogen peroxide staining, and SA quantification are described in detail in the Materials and Methods section of the Supplemental Information.

\section{RNA extraction, RNA-seq, data analysis, and gene expression analysis}

The experimental details and the relevant methods are described in the Materials and Methods section of the Supplementary Information.

\section{Supplementary Information}

Supplementary Information is available at https://doi.org/10.26508/lsa. 201800046.

\section{Acknowledgements}

This work was supported by the King Abdullah University of Science and Technology and Agence Nationale de la Recherche (ANR-2010-JCJC-1608). The Institute of Plant Sciences Paris-Saclay benefits from the support of the LabEx Saclay Plant Sciences (ANR-10-LABX-0040-SPS).

\section{Author Contributions}

AA Abulfaraj: conceptualization, data curation, formal analysis, validation, methodology, and writing-original draft, review, and editing.

K Mariappan: data curation, software, and formal analysis.

J Bigeard: conceptualization, methodology, and writing-review and editing.

P Manickam: visualization and methodology.

I Blilou: visualization, methodology, and writing-review and editing. X Guo: methodology.

S Albabili: supervision and methodology.

D Pflieger: conceptualization and writing-review and editing.

$\mathrm{H}$ Hirt: conceptualization, supervision, funding acquisition, project administration, and writing-review and editing.

N Rayapuram: conceptualization, data curation, formal analysis, supervision, validation, investigation, methodology, writing-original draft, project administration, and writing-review and editing.

\section{Conflict of Interest Statement}

The authors declare that they have no conflict of interest.

\section{References}

Acharya BR, Assmann SM (2009) Hormone interactions in stomatal function. Plant Mol Biol 69: 451-462. doi:10.1007/s11103-008-9427-0

Anderson P, Kedersha N (2008) Stress granules: The Tao of RNA triage. Trends Biochem Sci 33: 141-150. doi:10.1016/j.tibs.2007.12.003

Asai T, Tena G, Plotnikova J, Willmann MR, Chiu WL, Gomez-Gomez L, Boller T, Ausubel FM, Sheen J (2002) MAP kinase signalling cascade in Arabidopsis innate immunity. Nature 415: 977-983. doi:10.1038/415977a 
Baker B, Zambryski P, Staskawicz B, Dinesh-Kumar SP (1997) Signaling in plant-microbe interactions. Science 276: 726-733. doi:10.1126/ science. 276.5313 .726

Castello A, Fischer B, Frese CK, Horos R, Alleaume AM, Foehr S, Curk T, Krijgsveld J, Hentze MW (2016) Comprehensive identification of RNAbinding domains in human cells. Mol Cell 63: 696-710. doi:10.1016/j. molcel.2016.06.029

Chen YY, Lin YM, Chao TC, Wang JF, Liu AC, Ho Fl, Cheng CP (2009) Virusinduced gene silencing reveals the involvement of ethylene-, salicylic acid- and mitogen-activated protein kinase-related defense pathways in the resistance of tomato to bacterial wilt. Physiol Plant 136: 324-335. doi:10.1111/j.1399-3054.2009.01226.x

Dalton TP, Shertzer HG, Puga A (1999) Regulation of gene expression by reactive oxygen. Annu Rev Pharmacol Toxicol 39: 67-101. doi:10.1146/ annurev.pharmtox.39.1.67

Dangl JL, Jones JD (2001) Plant pathogens and integrated defence responses to infection. Nature 411: 826-833. doi:10.1038/35081161

Delaney TP, Uknes S, Vernooij B, Friedrich L, Weymann K, Negrotto D, Gaffney T, Gut-Rella M, Kessmann H, Ward E, et al (1994) A central role of salicylic acid in plant disease resistance. Science 266: 1247-1250. doi:10.1126/science.266.5188.1247

Ding Y, Shaholli D, Mou Z (2014) A large-scale genetic screen for mutants with altered salicylic acid accumulation in Arabidopsis. Front Plant Sci 5: 763. doi:10.3389/fpls.2014.00763

Felsenstein J (1985) Confidence limits on Phylogenies: An approach using the bootstrap. Evolution 39: 783-791. doi:10.2307/2408678

Frei dit Frey N, Garcia AV, Bigeard J, Zaag R, Bueso E, Garmier M, Pateyron S, de Tauzia-Moreau ML, Brunaud V, Balzergue S, et al (2014) Functional analysis of Arabidopsis immune-related MAPKs uncovers a role for MPK3 as negative regulator of inducible defences. Genome Biol 15: R87. doi:10.1186/gb-2014-15-6-r87

Geng X, Jin L, Shimada M, Kim MG, Mackey D (2014) The phytotoxin coronatine is a multifunctional component of the virulence armament of Pseudomonas syringae. Planta 240: 1149-1165. doi:10.1007/s00425014-2151-X

Jonas S, Izaurralde E (2013) The role of disordered protein regions in the assembly of decapping complexes and RNP granules. Genes Dev 27: 2628-2641. doi:10.1101/gad.227843.113

Kadota Y, Sklenar J, Derbyshire P, Stransfeld L, Asai S, Ntoukakis V, Jones JD, Shirasu K, Menke F, Jones A, et al (2014) Direct regulation of the NADPH oxidase RBOHD by the PRR-associated kinase BIK1 during plant immunity. Mol Cell 54: 43-55. doi:10.1016/j.molcel.2014.02.021

Katsafanas GC, Moss B (2007) Colocalization of transcription and translation within cytoplasmic poxvirus factories coordinates viral expression and subjugates host functions. Cell Host Microbe 2: 221-228. doi:10.1016/j.chom.2007.08.005

Kedersha N, Ivanov P, Anderson P (2013) Stress granules and cell signaling: More than just a passing phase? Trends Biochem Sci 38: 494-506. doi:10.1016/j.tibs.2013.07.004

Kedersha N, Panas MD, Achorn CA, Lyons S, Tisdale S, Hickman T, Thomas M, Lieberman J, McInerney GM, Ivanov P, et al (2016) G3BP-Caprin1-USP10 complexes mediate stress granule condensation and associate with 40S subunits. J Cell Biol 212: 845-860. doi:10.1083/jcb.201508028

Khokon AR, Okuma E, Hossain MA, Munemasa S, Uraji M, Nakamura Y, Mori IC, Murata Y (2011) Involvement of extracellular oxidative burst in salicylic acid-induced stomatal closure in Arabidopsis. Plant Cell Environ 34: 434-443. doi:10.1111/j.1365-3040.2010.02253.x

Kloek AP, Verbsky ML, Sharma SB, Schoelz JE, Vogel J, Klessig DF, Kunkel BN (2001) Resistance to Pseudomonas syringae conferred by an Arabidopsis thaliana coronatine-insensitive (coi1) mutation occurs through two distinct mechanisms. Plant J 26: 509-522. doi:10.1046/ j.1365-313x.2001.01050.x
Krapp S, Greiner E, Amin B, Sonnewald U, Krenz B (2017) The stress granule component G3BP is a novel interaction partner for the nuclear shuttle proteins of the nanovirus pea necrotic yellow dwarf virus and geminivirus abutilon mosaic virus. Virus Res 227: 6-14. doi:10.1016/j. virusres.2016.09.021

Kumar S, Stecher G, Tamura K (2016) MEGA7: Molecular evolutionary genetics analysis version 7.0 for bigger datasets. Mol Biol Evol 33: 1870-1874. doi:10.1093/molbev/msw054

Lamb CJ, Lawton MA, Dron M, Dixon RA (1989) Signals and transduction mechanisms for activation of plant defenses against microbial attack. Cell 56: 215-224. doi:10.1016/0092-8674(89)90894-5

Love AJ, Laval V, Geri C, Laird J, Tomos AD, Hooks MA, Milner JJ (2007) Components of Arabidopsis defense- and ethylene-signaling pathways regulate susceptibility to Cauliflower mosaic virus by restricting long-distance movement. Mol Plant Microbe Interact 20: 659-670. doi:10.1094/mpmi-20-6-0659

Mastrangelo AM, Marone D, Laido G, De Leonardis AM, De Vita P (2012) Alternative splicing: Enhancing ability to cope with stress via transcriptome plasticity. Plant Sci 185-186: 40-49. doi:10.1016/j. plantsci.2011.09.006

Melotto M, Underwood W, Koczan J, Nomura K, He SY (2006) Plant stomata function in innate immunity against bacterial invasion. Cell 126: 969-980. doi:10.1016/j.cell.2006.06.054

Mishina TE, Zeier J (2007) Pathogen-associated molecular pattern recognition rather than development of tissue necrosis contributes to bacterial induction of systemic acquired resistance in Arabidopsis. Plant J 50: 500-513. doi:10.1111/j.1365-313x.2007.03067.x

Mittler R, Vanderauwera S, Gollery M, Van Breusegem F (2004) Reactive oxygen gene network of plants. Trends Plant Sci 9: 490-498. doi:10.1016/j.tplants.2004.08.009

Moore JW, Loake GJ, Spoel SH (2011) Transcription dynamics in plant immunity. Plant Cell 23: 2809-2820. doi:10.1105/tpc.111.087346

Mori IC, Pinontoan R, Kawano T, Muto S (2001) Involvement of superoxide generation in salicylic acid-induced stomatal closure in Vicia faba. Plant Cell Physiol 42: 1383-1388. doi:10.1093/pcp/pce176

Navarro L, Zipfel C, Rowland O, Keller I, Robatzek S, Boller T, Jones JD (2004) The transcriptional innate immune response to flg22. Interplay and overlap with Avr gene-dependent defense responses and bacterial pathogenesis. Plant Physiol 135: 1113-1128. doi:10.1104/ pp.103.036749

O'Brien JA, Daudi A, Butt VS, Bolwell GP (2012) Reactive oxygen species and their role in plant defence and cell wall metabolism. Planta 236: 765-779. doi:10.1007/s00425-012-1696-9

Panas MD, Varjak M, Lulla A, Eng KE, Merits A, Karlsson Hedestam GB, McInerney GM (2012) Sequestration of G3BP coupled with efficient translation inhibits stress granules in Semliki Forest virus infection. Mol Biol Cell 23: 4701-4712. doi:10.1091/mbc.e12-08-0619

Ranf S, Eschen-Lippold L, Pecher P, Lee J, Scheel D (2011) Interplay between calcium signalling and early signalling elements during defence responses to microbe- or damage-associated molecular patterns. Plant J 68: 100-113. doi:10.1111/j.1365-313x.2011.04671.x

Reineke LC, Kedersha N, Langereis MA, van Kuppeveld FJ, Lloyd RE (2015) Stress granules regulate double-stranded RNA-dependent protein kinase activation through a complex containing G3BP1 and Caprin1. MBio 6: e02486. doi:10.1128/mbio.02486-14

Saitou N, Nei M (1987) The neighbor-joining method: A new method for reconstructing phylogenetic trees. Mol Biol Evol 4: 406-425. doi:10.1093/oxfordjournals.molbev.a040454

Sato M, Tsuda K, Wang L, Coller J, Watanabe Y, Glazebrook J, Katagiri F (2010) Network modeling reveals prevalent negative regulatory relationships between signaling sectors in Arabidopsis immune signaling. PLoS Pathog 6: e1001011. doi:10.1371/journal.ppat.1001011 
Singh P, Zimmerli L (2013) Lectin receptor kinases in plant innate immunity. Front Plant Sci 4: 124. doi:10.3389/fpls.2013.00124

Tourriere H, Chebli K, Zekri L, Courselaud B, Blanchard JM, Bertrand E, Tazi J (2003) The RasGAP-associated endoribonuclease G3BP assembles stress granules. I Cell Biol 160: 823-831. doi:10.1083/jcb.200212128

Tourriere H, Gallouzi IE, Chebli K, Capony JP, Mouaikel J, van der Geer P, Tazi J (2001) RasGAP-associated endoribonuclease G3Bp: Selective RNA degradation and phosphorylation-dependent localization. Mol Cell Biol 21: 7747-7760. doi:10.1128/mcb.21.22.7747-7760.2001

Tsuda K, Sato M, Glazebrook J, Cohen JD, Katagiri F (2008) Interplay between MAMP-triggered and SA-mediated defense responses. Plant J 53: 763-775. doi:10.1111/j.1365-313x.2007.03369.x

van Loon LC, Rep M, Pieterse CM (2006) Significance of inducible defenserelated proteins in infected plants. Annu Rev Phytopathol 44: 135-162. doi:10.1146/annurev.phyto.44.070505.143425

Wang L, Tsuda K, Truman W, Sato M, Nguyen le V, Katagiri F, Glazebrook J (2011) CBP60g and SARD1 play partially redundant critical roles in salicylic acid signaling. Plant J 67: 1029-1041. doi:10.1111/j.1365-313x.2011.04655.x

Wang Y, Bouwmeester K, van de Mortel JE, Shan W, Govers F (2013) A novel Arabidopsis-oomycete pathosystem: Differential interactions with Phytophthora capsici reveal a role for camalexin, indole glucosinolates and salicylic acid in defence. Plant Cell Environ 36: 1192-1203. doi:10.1111/pce.12052

White JP, Cardenas AM, Marissen WE, Lloyd RE (2007) Inhibition of cytoplasmic mRNA stress granule formation by a viral proteinase. Cell Host Microbe 2: 295-305. doi:10.1016/j.chom.2007.08.006

Xu J, Xie J, Yan C, Zou X, Ren D, Zhang S (2014) A chemical genetic approach demonstrates that MPK3/MPK6 activation and NADPH oxidasemediated oxidative burst are two independent signaling events in plant immunity. Plant J 77: 222-234. doi:10.1111/tpj.12382

Yang Y, Klessig DF (1996) Isolation and characterization of a tobacco mosaic virus-inducible myb oncogene homolog from tobacco. Proc Natl Acad Sci U S A 93: 14972-14977. doi:10.1073/pnas.93.25.14972

Yu X, Feng B, He P, Shan L (2017) From chaos to harmony: Responses and signaling upon microbial pattern recognition. Annu Rev Phytopathol 55: 109-137. doi:10.1146/annurev-phyto-080516-035649
Zeng Q, Chen JG, Ellis BE (2011a) AtMPK4 is required for male-specific meiotic cytokinesis in Arabidopsis. Plant / 67: 895-906. doi:10.1111/j.1365313x.2011.04642.x

Zeng W, Brutus A, Kremer JM, Withers JC, Gao X, Jones AD, He SY (2011b) A genetic screen reveals Arabidopsis stomatal and/or apoplastic defenses against Pseudomonas syringae pv. tomato DC3000. PLoS Pathog 7. doi:10.1371/journal.ppat.1002291

Zeng W, He SY (2010) A prominent role of the flagellin receptor FLAGELLINSENSING2 in mediating stomatal response to Pseudomonas syringae pv tomato DC3000 in Arabidopsis. Plant Physiol 153: 1188-1198. doi:10.1104/pp.110.157016

Zeng W, Melotto M, He SY (2010) Plant stomata: A checkpoint of host immunity and pathogen virulence. Curr Opin Biotechnol 21: 599-603. doi:10.1016/j.copbio.2010.05.006

Zhang Y, Xu S, Ding P, Wang D, Cheng YT, He J, Gao M, Xu F, Li Y, Zhu Z, et al (2010) Control of salicylic acid synthesis and systemic acquired resistance by two members of a plant-specific family of transcription factors. Proc Natl Acad Sci USA 107: 18220-18225. doi:10.1073/ pnas. 1005225107

Zheng XY, Spivey NW, Zeng W, Liu PP, Fu ZQ, Klessig DF, He SY, Dong X (2012) Coronatine promotes Pseudomonas syringae virulence in plants by activating a signaling cascade that inhibits salicylic acid accumulation. Cell Host Microbe 11: 587-596. doi:10.1016/j. chom.2012.04.014

Zipfel C, Kunze G, Chinchilla D, Caniard A, Jones JD, Boller T, Felix G (2006) Perception of the bacterial PAMP EF-Tu by the receptor EFR restricts Agrobacterium-mediated transformation. Cell 125: 749-760. doi:10.1016/j.cell.2006.03.037

Zipfel C, Robatzek S, Navarro L, Oakeley EJ, Jones JD, Felix G, Boller T (2004) Bacterial disease resistance in Arabidopsis through flagellin perception. Nature 428: 764-767. doi:10.1038/ nature02485

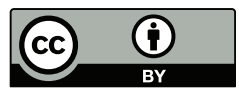

License: This article is available under a Creative Commons License (Attribution 4.0 International, as described at https://creativecommons.org/ licenses/by/4.0/). 